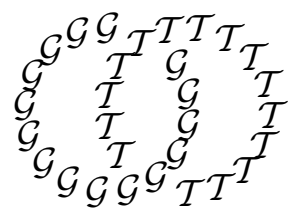

\title{
Logarithmic asymptotics of the genus zero Gromov-Witten invariants of the blown up plane
}

\author{
ILIA ITENBERG \\ ViATCHESLAV KHARLAMOV \\ EUGENII SHustin \\ (II and VK) Université Louis Pasteur et IRMA, 7, rue René Descartes, \\ 67084 Strasbourg Cedex, France \\ and \\ (ES) School of Mathematical Sciences, Tel Aviv University \\ Ramat Aviv, 69978 Tel Aviv, Israel \\ Email: itenberg@math.u-strasbg.fr, kharlam@math.u-strasbg.fr \\ shustin@post.tau.ac.il
}

\begin{abstract}
We study the growth of the genus zero Gromov-Witten invariants $G W_{n D}$ of the projective plane $P_{k}^{2}$ blown up at $k$ points (where $D$ is a class in the second homology group of $P_{k}^{2}$ ). We prove that, under some natural restrictions on $D$, the sequence $\log G W_{n D}$ is equivalent to $\lambda n \log n$, where $\lambda=D \cdot c_{1}\left(P_{k}^{2}\right)$.
\end{abstract}

\section{AMS Classification numbers Primary: 14N35}

Secondary: $14 \mathrm{~J} 26,53 \mathrm{D} 45$

Keywords: Gromov-Witten invariants, rational and ruled algebraic surfaces, rational and ruled symplectic 4-manifolds, tropical enumerative geometry

Proposed: Yasha Eliashberg

Seconded: Leonid Polterovich, Simon Donaldson
Received: 30 December 2004

Accepted: 25 March 2005 


\section{Introduction}

In this note we treat the asymptotic behavior of the genus zero Gromov-Witten invariants on 4-dimensional symplectic manifolds. In this setting such an invariant can be seen as a count of connected rational $J$-holomorphic curves in a given homology class under a choice of a generic tamed almost complex structure, see, for example, [12.

Our interest to logarithmic asymptotics is motivated by a comparison of Gromov-Witten invariants with their real analogs introduced by J-Y Welschinger (see [5] and the conjecture in Section 4) and by a relation of the logarithmic asymptotics with the convergency properties of the Gromov-Witten potential (see [5]).

As is known, already the existence of homology classes with a nontrivial invariant which are distinct from the homology classes of $(-1)$-curves is a very restrictive condition. It implies that the 4-dimensional symplectic manifold in question is a blow-up of a rational or ruled manifold (precise statements, details, and references can be found in [12, Section 9.4). We exclude irrational ruled manifolds (that is, symplectic $S^{2}$-bundles over Riemann surfaces of genus $g>0$ ) since they have only one homology class with a nontrivial invariant, the class represented by the fiber. Furthermore, since the Gromov-Witten invariants are preserved under variations of the symplectic structure, for the study of their asymptotic properties it is sufficient to consider the product of complex projective lines, $\mathbb{P}^{1} \times \mathbb{P}^{1}$, and the blow-ups of the complex projective plane.

Let us denote by $\mathbb{P}_{k}^{2}$ the complex projective plane $\mathbb{P}^{2}$ blown up at $k$ points. Pick a homological class $D$ in $H_{2}\left(\mathbb{P}_{k}^{2} ; \mathbb{Z}\right)$ such that the Gromov-Witten invariant $G W_{D}\left(\mathbb{P}_{k}^{2}\right)$ is non-zero, and either $D \cdot c_{1}\left(\mathbb{P}_{k}^{2}\right)>2$, or $D \cdot c_{1}\left(\mathbb{P}_{k}^{2}\right)=2$ and $D^{2}>0$. Under the above hypotheses on $D$, the Gromov-Witten invariants of $n D, n \geq 1$, are enumerative, that is, the invariant $\mathrm{GW}_{n D}\left(\mathbb{P}_{k}^{2}\right), n \geq 1$, is equal to the number $N_{n D}\left(\mathbb{P}_{k}^{2}\right)$ of immersed irreducible rational curves passing through $n D \cdot c_{1}\left(\mathbb{P}_{k}^{2}\right)-1$ given generic points in $\mathbb{P}_{k}^{2}$ under the additional assumption that the blown up points are also generic, see [2].

In the case of $\mathbb{P}_{0}^{2}=\mathbb{P}^{2}$ the Kontsevich recursive formula for $N_{n L}\left(\mathbb{P}^{2}\right)$ [ being a line in $\mathbb{P}^{2}$ ) allows one to get successive values of these invariants and to find their asymptotics. In particular, one has $\log N_{n L}\left(\mathbb{P}^{2}\right)=3 n \log n+O(n)$ as $n \rightarrow+\infty$ (see [1]). There exist recursive formulas for the Del Pezzo surfaces, see [9], and for $\mathbb{P}_{k}^{2}$ with any $k$, see [2]. However, these formulas are not easy to analyze specially for large $k$. In [5] working with the corresponding counts of real curves we observed, by means of Mikhalkin's theorem [13 14] (see also [16]) 
on the enumeration of nodal curves on toric surfaces via lattice paths in convex lattice polygons, that the relation

$$
\log N_{n D}(\Sigma)=\lambda n \log n+O(n), \quad \lambda=D \cdot c_{1}(\Sigma),
$$

holds for any ample divisor $D$ on a toric Del Pezzo surface $\Sigma$, in particular, on the plane with blown up one, two, or three points, and on $\mathbb{P}^{1} \times \mathbb{P}^{1}$.

The present note is devoted to a proof of the following theorem.

Theorem 1 Let $\mathbb{P}_{k}^{2}$ be the plane blown up at $k \geq 1$ points, and $D \in H_{2}\left(\mathbb{P}_{k}^{2} ; \mathbb{Z}\right)$ a homology class such that $G W_{D}\left(\mathbb{P}_{k}^{2}\right) \neq 0$ and either $D \cdot c_{1}\left(\mathbb{P}_{k}^{2}\right)>2$, or $D$. $c_{1}\left(\mathbb{P}_{k}^{2}\right)=2$ and $D^{2}>0$. Then

$$
\log G W_{n D}\left(\mathbb{P}_{k}^{2}\right)=\lambda n \log n+O(n), \quad \lambda=D \cdot c_{1}\left(\mathbb{P}_{k}^{2}\right) .
$$

As a consequence we get the following enumerative statement.

Corollary 1 Let $\mathbb{P}_{k}^{2}$ be the plane blown up at $k \geq 1$ generic points, and $D \in H_{2}\left(\mathbb{P}_{k}^{2} ; \mathbb{Z}\right)$ is as in Theorem 11. Then

$$
\log N_{n D}\left(\mathbb{P}_{k}^{2}\right)=\lambda n \log n+O(n), \quad \lambda=D \cdot c_{1}\left(\mathbb{P}_{k}^{2}\right) .
$$

Furthermore, if $k \leq 9$, then (2) holds for any ample divisor $D$ on $\mathbb{P}_{k}^{2}$.

Let us notice that the hypotheses of Theorem 1 are in a sense optimal. For example, $G W_{n D}=0$ if $n \geq 2$ and $D$ is an embedded curve with $D \cdot c_{1} \leq 2$.

\section{Rational curves on rational geometrically ruled surfaces}

Here we prove two auxiliary statements.

Lemma 1 Let $\Sigma_{s}, s>0$, be a rational geometrically ruled surface with the exceptional section $E, E^{2}=-s$, and a fibre $F$. Then,

$$
\log N_{n(s F+E)}\left(\Sigma_{s}\right) \geq(s+2) n \log n+O(n) .
$$

Proof We follow the ideas of the proof of Lemma 5 in $[5]$.

First, we observe that the case $s=1$ corresponds to curves on $\Sigma=\mathbb{P}_{1}^{2}$ disjoint from $E$, and since $s+2=(s F+E) \cdot c_{1}(\Sigma)$, this case is settled in Theorem 3 of [5] applied to $\mathbb{P}^{2}$. Then, we assume that $s \geq 2$ and prove the inequality

$$
N_{n(s F+E)}\left(\Sigma_{s}\right) \geq n ! \cdot N_{n((s-1) F+E)}\left(\Sigma_{s-1}\right),
$$



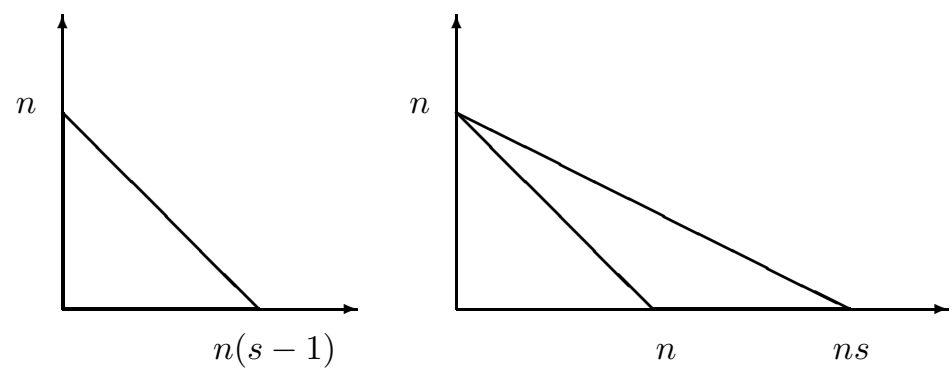

Figure 1: Correspondence between rational curves on $\Sigma_{s-1}$ and $\Sigma_{s}$

which immediately implies

$$
\log N_{n(s F+E)}\left(\Sigma_{s}\right) \geq \log N_{n L}\left(\mathbb{P}^{2}\right)+(s-1) \log n !,
$$

and hence the inequality (31) in view of $\log N_{n L}\left(\mathbb{P}^{2}\right)=3 n \log n+O(n)$.

To prove (4), notice that the number $N_{n((s-1) F+E)}\left(\Sigma_{s-1}\right)$ of rational curves in the linear system $|n((s-1) F+E)|$ passing through $(s+1) n-1$ generic points in $\Sigma_{s-1}$ can be viewed as the number of rational curves in the linear system $\mid n(s F+$ $E) \mid$ on $\Sigma_{s}$ which pass through $(s+1) n-1$ generic points and have an ordinary $n$-fold singularity at some fixed point $z$ (this correspondence is provided by the birational transformation $\Sigma_{s-1} \rightarrow \Sigma_{s}$ given in suitable affine toric coordinates $x, y$ in $\Sigma_{s-1}$ and $u, v$ in $\Sigma_{s}$ by $u=x, v=x y$; the correspondence reflects an affine transformation of Newton polygons as shown in Figure 11). Choose now generic points in a small neighborhood of $z$. The argument of the proof of Lemma 5 in [5] confirms that any rational curve $C \in|n(s F+E)|$ as above can be deformed inside the class of rational curves passing through the initial $(s+1) n-1$ fixed points, so that the $n$ local branches of $C$ at $z$ freely move in transverse directions, and hence can be traced through the newly chosen $n$ fixed points in an arbitrary order. Thus, (4) follows.

Remark 1 Lemma 1 can also be proved using the tropical count. The proof is completely similar to the proof of Theorem 3 (case of $\mathbb{P}^{2}$ ) in [5]. One should just adapt the corresponding lattice path constructed in [5] to a triangle representing the linear system $|n(s F+E)|$ on $\Sigma_{s}$.

Lemma 2 Fix an integer $s \geq 1$. Then, there exists an integer sequence $\left(T_{n}\right)$ which verifies the following properties: $\log T_{n}=(s+2) n \log n+O(n)$ and for 
any $n$ there is a generic collection $z_{1}, \ldots z_{(s+2) n-1}$ of $(s+2) n-1$ points in $\Sigma_{s}$ such that among the rational curves belonging to the linear system $|n(s F+E)|$ and passing through $z_{i}, i=1, \ldots,(s+2) n-1$, at least $T_{n}$ curves have only ordinary nodes as singular points and intersect each other transversally outside of the points $z_{i}, i=1, \ldots,(s+2) n-1$.

Proof For $s=1$ the statement on ordinary nodes is classical and holds for all the interpolating curves in the linear system (see, for example [15] for a modern exposition). For $s>1$ one can apply the construction described in the proof of Lemma 1 and observe that it preserves the statement on ordinary nodes. To eliminate one by one eventual non-transversal intersections of interpolating rational curves outside of the chosen points, it suffices to move one of the chosen points along an interpolating curve having a non-transversal intersection with another curve.

\section{Proof of Theorem 1 and Corollary 1}

Proof of Theorem 1 Let us first note that under the hypotheses on $D$ made in Theorem 11, the Gromov-Witten invariants are enumerative. More precisely, deforming the complex structure of $\mathbb{P}_{k}^{2}$ to a generic almost complex one we observe, first, that, due to $G W_{D}\left(\mathbb{P}_{k}^{2}\right) \neq 0$ and the stability of the exceptional divisors $E_{1}, \ldots, E_{k}$ of $\mathbb{P}_{k}^{2} \rightarrow \mathbb{P}^{2}$, the non-negativity of intersections, $D \cdot E_{i} \geq 0$, holds, and then, by Theorem 4.1 in [2], we obtain that due to $D \cdot c_{1}\left(\mathbb{P}_{k}^{2}\right)>1$, for a generic choice of the $k$ blown-up points the number $\mathrm{GW}_{n D}\left(\mathbb{P}_{k}^{2}\right), n \geq 1$, is equal to the number $N_{n D}\left(\mathbb{P}_{k}^{2}\right)$ of immersed irreducible rational curves passing through $n D \cdot c_{1}\left(\mathbb{P}_{k}^{2}\right)-1$ given generic points in $\mathbb{P}_{k}^{2}$.

The upper bound, $\log N_{n D}\left(\mathbb{P}_{k}^{2}\right) \leq\left(D \cdot c_{1}\left(\mathbb{P}_{k}^{2}\right)\right) n \log n+O(n)$, is given by Lemma 5 in [5].

To prove the lower bound, assume, first, that $D \cdot c_{1}\left(\mathbb{P}_{k}^{2}\right)>2$. By [2], Theorem 4.1, we can represent $D$ by an immersion $\varphi: \mathbb{P}^{1} \rightarrow \mathbb{P}_{k}^{2}$. We consider the bundle $\mathcal{N}_{\mathbb{P}^{1}}=\varphi^{*}\left(\mathcal{T} \mathbb{P}_{k}^{2}\right) / \mathcal{T} \mathbb{P}^{1}$ over $\mathbb{P}^{1}$ and compactify it into the rational ruled surface $X=\operatorname{Proj}\left(\mathcal{N}_{\mathbb{P}^{1}} \oplus \mathcal{O}_{\mathbb{P}^{1}}\right)$. Pick Kähler structures on $\mathbb{P}_{k}^{2}$ and $X$ with the same periods on, respectively, $D$ in $\mathbb{P}_{k}^{2}$ and $\mathbb{P}^{1}$ in $X$, and fix a symplectic immersion $\Phi$ of a small neighborhood $\mathfrak{N}\left(\mathbb{P}^{1}\right) \subset X$ into $\mathbb{P}_{k}^{2}$ which extends $\varphi$. Such an immersion $\Phi$ exists due to the symplectic neighborhood theorem (see, for example, [1], Theorem 3.30) Notice that $D \cdot c_{1}\left(\mathbb{P}_{k}^{2}\right)=\mathbb{P}^{1} \cdot c_{1}(X)$. Therefore, applying Lemma 2 to the linear system $\left|n \mathbb{P}^{1}\right|$ we find in it $T_{n}, T_{n}=D$. $c_{1}\left(\mathbb{P}_{k}^{2}\right) n \log n+O(n)$, immersed rational curves which pass through $D \cdot c_{1}\left(\mathbb{P}_{k}^{2}\right)-1$ 
generic fixed points, have only ordinary nodes as singular points, and intersect transversally outside of the fixed points. Choose 0 and $\infty$ in $\mathbb{P}^{1}$ so that the fibers over them are transversal to each of these $T_{n}$ curves $C_{i}$ and do not contain any of the fixed $D \cdot c_{1}\left(\mathbb{P}_{k}^{2}\right)-1$ points. Now by vertical and horizontal (toric) rescaling in $X$ we can make all the curves $C_{i}$ to be $C^{0}$-close to $\mathbb{P}^{1}$ and, moreover, $C^{1}$-close to it outside an arbitrary small neighborhood of $0 \in \mathbb{P}^{1}$. As a consequence, we get $T_{n}$ immersed symplectic surfaces $\Phi\left(C_{i}\right)$ which pass through some common $D \cdot c_{1}\left(\mathbb{P}_{k}^{2}\right)-1$ points, have only ordinary nodes as singular points, and are transversal to each other outside of the common $D \cdot c_{1}\left(\mathbb{P}_{k}^{2}\right)-1$ points. Proceeding as in 10, Lemma 3.2, we construct a tamed almost complex structure $J$ on $\mathbb{P}_{k}^{2}$ for which all the surfaces $\Phi\left(C_{i}\right)$ are $J$-holomorphic (we start from neighborhoods of the common points, where we retrieve a suitable almost complex structure from $X$ ). Due to [7, the constructed $J$-holomorphic curves represent discrete regular solutions of the interpolating problem. Thus, to get the desired below bound it remains to notice that, as it follows from [7] and 6] (Corollaries 1.6 and 2.7), the space of generic almost complex structures is connected and dense, and each regular solution counts for +1 .

In the remaining case, $D \cdot c_{1}\left(\mathbb{P}_{k}^{2}\right)=2$ and $D^{2}>0$, the conditions $D^{2}>0$ and $G W_{D}\left(\mathbb{P}_{k}^{2}\right) \neq 0$ imply, by the standard gluing argument, that $G W_{2 D}\left(\mathbb{P}_{k}^{2}\right) \neq 0$. Therefore, the preceding case applies to $D^{\prime}=2 D$ and the lower bound now follows from the monotonicity relation

$$
N_{(n+1) D}\left(\mathbb{P}_{k}^{2}\right) \geq N_{n D}\left(\mathbb{P}_{k}^{2}\right), n \geq 1 .
$$

To get (5) we use again the gluing of rational curves. Namely, we construct an injective map from the set of rational curves in $|n D|$ passing through $2 n-1=$ $n D \cdot c_{1}\left(\mathbb{P}_{k}^{2}\right)-1$ fixed generic points to the set of rational curves in $|(n+1) D|$ passing through $2 n+1$ generic points. Pick $2 n$ generic points $p_{i}, i=1, \ldots, 2 n$, in $\mathbb{P}_{k}^{2}$, and a rational curve $C_{1} \in|D|$ passing through $p_{2 n}$. We can assume that for any curve $C$ chosen among the rational curves belonging to $|n D|$ and passing through $p_{1}, \ldots, p_{2 n-1}$, there is a point $z_{C} \in C \cap C_{1}$ which is singular neither for $C$ no for $C_{1}$ and where the curves $C$ and $C_{1}$ intersect transversally. Pick a generic point $p^{\prime} \in C_{1}$ and a point $p_{2 n+1} \notin C_{1}$ in a small neighborhood of $p^{\prime}$. Then, there exists a one-parameter deformation of $C \cup C_{1}$ consisting of rational curves in $|(n+1) D|$ and such that the point $z_{C}$ smoothes out and the points $p_{1}, \ldots, p_{2 n}$ remain fixed (see, for example, 3], Proposition 5.2, or [8], Ch. II, Theorem 7.6). This family sweeps a neighborhood of $p^{\prime}$, and hence we obtain a rational curve $C^{\prime} \in|(n+1) D|$ passing through $p_{1}, \ldots, p_{2 n-1}, p_{2 n}, p_{2 n+1}$.

Remark 2 The relation (1) of the statement of Theorem 11 is also valid for a homology class $D \in H_{2}\left(\mathbb{P}_{k}^{2} ; \mathbb{Z}\right)$ such that $G W_{D}\left(\mathbb{P}_{k}^{2}\right)>1, D \cdot c_{1}\left(\mathbb{P}_{k}^{2}\right)=1$, and 
$D^{2}>0$. Indeed, putting $D^{\prime}=2 D$, we have $D^{\prime} \cdot c_{1}\left(\mathbb{P}_{k}^{2}\right)=2$ and $\left(D^{\prime}\right)^{2}>0$. By [2, Theorem 4.1, we can find in the linear system $|D|$ two distinct immersed rational curves. Then, deforming these curves as in the proof of Theorem 1] we get $G W_{D^{\prime}}\left(\mathbb{P}_{k}^{2}\right)>0$. Thus, the relation (1) holds for $D^{\prime}$, and we can prove this relation for $D$ using the inequality (5) as is done in the proof of Theorem 1 .

Proof of Corollary 1 The first statement immediately follows from Theorem 1 and the equality $\mathrm{GW}_{n D}\left(\mathbb{P}_{k}^{2}\right)=N_{n D}\left(\mathbb{P}_{k}^{2}\right)$ explained in the beginning of the proof of Theorem 1.

To prove the second statement, we observe first that any ample divisor $D$ on $\mathbb{P}_{k}^{2}$, $k \leq 9$, is represented by a nodal rational curve. For $k=1$ or 2 this is trivial. For $3 \leq k \leq 9$ this follows from Theorem 5.2 in [4], which states the existence of rational nodal curves in certain linear systems in $\mathbb{P}_{k}^{2}$. Indeed, given an expansion $D=d L-d_{1} E_{1}-\ldots-d_{k} E_{k}$ for any base $\left(L, E_{1}, \ldots, E_{k}\right)$ of $\operatorname{Pic}\left(\mathbb{P}_{k}^{2}\right)$ satisfying $L^{2}=-E_{1}^{2}=\ldots=-E_{k}^{2}=1$, the ampleness of $D$ yields that $d, d_{1}, \ldots, d_{k}>0$. Furthermore, by base changes in $\operatorname{Pic}\left(\mathbb{P}_{k}^{2}\right)$ induced by Cremona transformations (see 4, section 5.1), we can achieve $d \geq \max _{i \neq j \neq l}\left(d_{i}+d_{j}+d_{l}\right)$, which is the minimality condition of Theorem 5.2 in [4. At last, the remaining condition of this theorem, $3 d>d_{1}+\ldots+d_{k}$ (which can be also written as $D \cdot c_{1}\left(\mathbb{P}_{k}^{2}\right)>0$ ), follows from the positivity of intersection with the strict transform of a plane cubic passing through the blown-up points.

Since the existence of a nodal rational curve in $|D|$ implies $G W_{D}\left(\mathbb{P}_{k}^{2}\right) \neq 0$, and since, in addition, $D^{2}>0$ for any ample $D$, the second statement of the corollary is proved for ample divisors $D$ satisfying $D \cdot c_{1}\left(\mathbb{P}_{k}^{2}\right) \geq 2$.

Now let us consider the case $D \cdot c_{1}\left(\mathbb{P}_{k}^{2}\right)=1$, and put $D^{\prime}=2 D$. We have $D^{\prime} \cdot c_{1}\left(\mathbb{P}_{k}^{2}\right)=2$ and $\left(D^{\prime}\right)^{2}>0$, and once more by Theorem 5.2 in [4] we get $G W_{D^{\prime}}\left(\mathbb{P}_{k}^{2}\right) \neq 0$. Hence, the relation (2) holds for $D^{\prime}$, and finally we deduce this relation for $D$ using the inequality (5) as is done in the proof of Theorem 1

\section{Welschinger invariants of real rational surfaces}

Recall that the Welschinger invariants depend not only on a homology class, but also on a number of non-real points in a real configuration of points (see 17, 18, for the definition and properties of the Welschinger invariants). Denote by $W_{n D}\left(\mathbb{P}_{k}^{2}\right)$ the Welschinger invariant which counts, with weights \pm 1 , the real rational curves belonging to the linear system $|n D|$ and passing through $n D \cdot c_{1}\left(\mathbb{P}_{k}^{2}\right)-1$ given generic real points in $\mathbb{P}_{k}^{2}$. As is proved in $[\underline{5}$, in the cases 
$k=1,2,3$, the same relation as (10) holds for $W_{n D}\left(\mathbb{P}_{k}^{2}\right)\left(\right.$ instead of $\left.\mathrm{GW}_{n D}\left(\mathbb{P}_{k}^{2}\right)\right)$. This motivates the following conjecture.

Conjecture 1 Assume that $\mathbb{P}_{k}^{2}$ is obtained from $\mathbb{P}^{2}$ by blowing up $k$ generic real points and is equipped with its natural real structure. Let $D \subset \mathbb{P}_{k}^{2}$ be a real ample divisor. Then, the Welschinger invariants $W_{n D}\left(\mathbb{P}_{k}^{2}\right)$ satisfy the relation

$$
\lim _{n \rightarrow+\infty} \frac{\log W_{n D}\left(\mathbb{P}_{k}^{2}\right)}{n \log n}=D \cdot c_{1}\left(\mathbb{P}_{k}^{2}\right) .
$$

One could try to prove Conjecture 1using the same construction as in the proof of Theorem 1. However, this approach does not give immediately the result, since a real regular solution to the interpolation problem contributes \pm 1 to the Welschinger invariant. Thus, to get an asymptotic lower bound, it is not enough to present an appropriate number of interpolating real rational curves.

\section{Acknowledgements}

The idea of this work came to the authors during their stay at the Max-PlanckInstitut für Mathematik in Bonn, and we thank this institution for the hospitality and excellent work conditions. The first and the second authors are members of Research Training Network RAAG CT-2001-00271. The third author was supported by the Israel Science Foundation grant no. 465/04 and by the Nermann-Minkowski-Minerva Center for Geometry at the Tel Aviv University.

We are also grateful to the referee for useful remarks.

\section{References}

[1] P Di Francesco,C Itzykson, Quantum intersection rings, from: "The moduli space of curves (Texel Island, 1994)", Progr. Math. 129, Birkhäuser, Boston, MA (1995) 81-148 MathReview

[2] L Göttsche, A Pandharipande, The quantum cohomology of blow-ups of $\mathbb{P}^{2}$ and enumerative geometry, J. Differential Geom. 48 (1998) 61-90 MathReview

[3] G-M Greuel, U Karras, Families of varieties with prescribed singularities, Compositio Math. 69 (1989) 83-110 MathReview

[4] G-M Greuel, C Lossen, E Shustin, Geometry of families of nodal curves on the blown up projective plane, Trans. Amer. Math. Soc. 350 (1998), no. 1, 251-274 MathReview 
[5] I Itenberg, V Kharlamov, E Shustin, Logarithmic equivalence of Welschinger and Gromov-Witten invariants, Russian Math. Surveys 59 (2004) 1093-1116

[6] S M Ivashkovich, V V Shevchishin, Deformations of non-compact complex curves and envelopes of meromorphy of spheres, Mat. Sb. 189 (1998) 23-60 (Russian); translation in Sb. Math. 189 (1998) 1335-1359 MathReview

[7] H Hofer, V Lizan, J-C Sikorav, On genericity for holomorphic curves in four-dimensional almost-complex manifolds, J. Geom. Anal. 7 (1998) 149-159 MathReview

[8] J Kollár, Rational curves on algebraic varieties, Ergebnisse der Mathematik und ihrer Grenzgebiete 32, Springer-Verlag, Berlin (1996) MathReview

[9] M Kontsevich, Yu Manin, Gromov-Witten classes, quantum cohomology and enumerative geometry, Commun. Math. Phys. 164 (1994) 525-562 MathReview

[10] D McDuff, Immersed spheres in symplectic 4-manifolds, Ann. Inst. Fourier 42 (1992) 369-392 MathReview

[11] D McDuff, D Salamon, Introduction to Symplectic Topology, 2nd edition, Oxford Mathematical Monographs, Oxford Univ. Press, Oxford (1998) MathReview

[12] D McDuff, D Salamon, J-holomorphic Curves and Symplectic Topology, Amer. Math. Soc. Colloquium Publications 52, Amer. Math. Soc. Providence RI (2004) MathReview

[13] G Mikhalkin, Counting curves via the lattice paths in polygons, C. R. Acad. Sci. Paris, Sér. I, 336 (2003) 629-634 MathReview

[14] G Mikhalkin, Enumerative tropical algebraic geometry in $\mathbb{R}^{2}$, J. Amer. Math. Soc. 18 (2005), 313-377

[15] A Nobile, On specialization of curves I, Trans. Amer. Math. Soc. 282 (1984) 739-748 MathReview

[16] E Shustin, A tropical approach to enumerative geometry, Algebra Anal. 17 (2005) 170-214

[17] J-Y Welschinger, Invariants of real rational symplectic 4-manifolds and lower bounds in real enumerative geometry, C. R. Acad. Sci. Paris, Sér. I, 336 (2003) 341-344 MathReview

[18] J-Y Welschinger, Invariants of real symplectic 4-manifolds and lower bounds in real enumerative geometry, Invent. Math. to appear, arXiv:math.AG/0303145 